\section{El significado del capital social "individual" en diabéticos receptores de cuidado en un contexto urbano colombiano}

\author{
The meaning of "individual" social capital for \\ diabetics receiving care in a Colombian city
}

\author{
O significado do capital social "individual" em diabéticos \\ que precisam de cuidados dentro de um contexto \\ urbano colombiano
}

\author{
1 Facultad Nacional de \\ Salud Pública, Universidad \\ de Antioquia, Medellín, \\ Colombia. \\ 2 Instituto de Evaluación de \\ Tecnologías en Salud, Bogotá \\ DC, Colombia. \\ Correspondencia \\ E. Martínez-Herrera \\ Facultad Nacional de Salud \\ Pública, Universidad de \\ Antioquia. \\ Calle 62 No. 52-59, Medellín, \\ Antioquia, Colombia. \\ zenitram34@gmail.com
}

\begin{abstract}
The aim of this study was to understand the meaning of social capital in relation to type 2 diabetes according to gender, within an urban setting in Colombia, based on a qualitative design for symbolic interactionism. Twenty-four women and 16 men with diabetes, family members, and healthcare personnel participated in six focus groups. A total of 850 codes emerged that comprised a set of 142 codes for ego, alter, and alter ego. Three categories and 20 subcategories were identified for the "coding paradigm design". The meaning differed between men and women. Social ties in social networks, created daily through trust and solidarity for care were valued differently due to the social experiences and events resulting from self-confidence, self-efficacy for social support, and mainly selfesteem vis-à-vis management and control of the disease. An individual's social resources are reified for the management and care of the disease as a strategy to mitigate health inequalities.
\end{abstract}

Type 2 Diabetes Mellitus; Social Networking; Health Inequalities
Eliana Martínez-Herrera 1 Ornella Moreno-Mattar 2 Robert V. H. Dover 1

\section{Resumen}

Comprender el significado del capital social de la diabetes tipo 2 según género, dentro un contexto urbano colombiano. Investigación cualitativa del interaccionismo simbólico. 25 mujeres y 16 hombres, diabéticos, familiares, vecinos y personal asistencial participaron en seis grupos focales. Emergieron 850 códigos que se integraron en un set de 142 códigos de códigos para el ego, el alter $y$ alter ego. Tres categorías y veinte subcategorías fueron identificadas para el diseño del "paradigma de la codificación". El significado no es igual para hombres y mujeres. Los vínculos sociales de las redes sociales, creados cotidianamente por la confianza y la solidaridad para el cuidado, son valorados de manera diferente, debido a experiencias y hechos sociales resultantes de la autoconfianza, la autoeficacia para el apoyo social principalmente y, la autoestima frente al manejo y control de la enfermedad. Los recursos sociales de un individuo son reificados para el manejo y cuidado de la enfermedad como estrategia para disminuir las inequidades en salud.

Diabetes Mellitus Tipo 2; Red Social; Desigualdades en la Salud 


\section{Introducción}

La condición de cronicidad de la diabetes determina el manejo clínico y social de la enfermedad, impactando no sólo en la calidad de vida de los pacientes, sino también en los sistemas productivos de los países 1. Ante tal condición, un diabético descubre elementos en el entorno para ordenar las acciones cotidianas hacia el intercambio social, construye conceptos mentales ${ }^{2}$, y toma decisiones de manera individual sobre el padecimiento, que con el tiempo son reflejados en patrones culturales compartidos frente al cuidado de la enfermedad por grupos en condiciones similares 3 .

Pese a que existen muchos esquemas de prevención reportados en la literatura para el seguimiento de los estilos de vida, de los factores personales y familiares que inciden en la aparición de complicaciones y cuidado primario de los diabéticos 4 , pocos de ellos han incluido de manera novedosa, la perspectiva del capital social "individual" en las políticas de salud pública, así como las oportunidades que las redes sociales proporcionan ampliamente en la vida de las personas para afrontar la enfermedad.

A pesar de que el capital social produce sobre la estructura social hechos cambiantes para la construcción de respuestas y de nuevos efectos sociales sobre las personas, existen aproximaciones teóricas diferentes, controvertidas $y$, antagónicas que limitan su uso, debido entre otros motivos por el interés político, económico y social en el momento histórico en que se concibe el concepto pero, además, por su aplicación ahistórica 5,6

En la actualidad, la mayor evidencia empírica de las aplicaciones del uso del concepto en salud pública se encuentra en los estudios de capital social "contextual" 7,8, más que de capital social "individual" 9,10; los resultados de ellos evidencian la acción colectiva para el bienestar 11 social, incluso actuando como recursos de los individuos en la vinculación con valores como la confianza social, los derivados de normas de reciprocidad, la ayuda mutua, entre otros y, su relación con la salud 12 .

Aun cuando, las recomendaciones internacionales se interesan en promover la comprensión de los determinantes sociales, económicos y culturales del proceso salud-enfermedad como la estrategia clave para mejorar las acciones de salud pública 13 , se cuenta con pocos estudios que identifican el rol del capital social "individual" para lograr efectos en el control y manejo de la diabetes tipo 2. Es de resaltar que este determinante de la salud puede ser analizado desde el nivel macro, como cohesión social, hasta el nivel micro como, por ejemplo, apoyo social y en su nivel intermedio como soporte social propiamente dicho, de manera que no sólo se refiere a las causas de las causas únicamente estructurales, sino también las que le corresponden al individuo y, por tanto, la reducción de los daños a lo largo del proceso de salud enfermedad con padecimientos crónicos degenerativos. La diabetes tipo 2 es compleja y debe promoverse mediante soluciones altamente integradoras de tipo social, comunitarias e individuales.

Ahora bien, con la pragmática circunscrita a la cultura y a los lugares 14 , interesa interpretar a través de la argumentación 15, el uso de los recursos del capital social “individual” de los pacientes en el manejo y control de la diabetes tipo 2 para, primero, darlo a conocer a los formuladores y tomadores de decisión de políticas públicas en salud en el evento de incluirlo en las estrategias de mitigación de la enfermedad y, segundo, a los cuidadores y receptores del cuidado para que le identifiquen como un constructo que de ser fomentado en su práctica diaria 16 e interfiera en el desarrollo de la enfermedad.

Con la construcción del significado de capital social "individual" se reconocen nuevos aspectos que deben ser incorporados en las formas de intervención psíquicas y sociales, nuevos diálogos para la creación de estrategias entre profesionales de la salud y pacientes, debido a las características de la relación y las diversas formas de entender el cuidado, pero aún más valioso, tiene todo que ver con la comprensión que tienen las interacciones sociales en contextos institucionales y comunitarios, interacciones que manifiestan realidades sociales diferentes, debido a los cambios actitudinales del individuo en el manejo y control de la enfermedad, los cuales dependen de los recursos propios del diabético, de las acciones e interacciones que se desarrollan bajo la coacción social de estructuras sociales 17 entre las personas sujetas o no al padecimiento.

En el estudio, las interacciones están provistas de elementos analizados a partir de las emociones de los participantes sobre prácticas del cuidado, aceptación y manejo de la enfermedad y en conjunto, relaciones e intercambios que integran las experiencias en el proceso del cuidado de quien padece la diabetes tipo 2 , convive con ella y se ha preparado para soportarla en contextos inequitativos e injustos, como lo es el de ciudades colombianas con más de tres millones de habitantes, con más del $70 \%$ de sus habitantes localizados en las zonas urbanas, caracterizado históricamente por profundas fisuras sociales, debidas entre otras dificultades, a la violencia y las consecuentes inequidades sociales, culturales y económicas 18 . 


\section{Métodos}

Mediante un estudio cualitativo descriptivointerpretativo, basado en los postulados del interaccionismo simbólico 19 se conformaron seis grupos focales con 40 personas diabéticos, familiares, vecinos y personal asistencial, pertenecientes a centros de salud público y privados, 25 mujeres y 16 hombres, entre los 29 y 73 años de edad, residentes en Medellín, Colombia, durante el 2011 para esclarecer el significado del capital social 20,21 en la diabetes tipo 2 .

El muestreo teórico se realizó acorde a las necesidades de conformación de los grupos focales; para los diabéticos receptores del cuidado se usó la estrategia de búsqueda mediante revisión de historia clínica de listados de pacientes adscritos a los centros de salud de una aseguradora privada y de la red pública de prestación de servicios. Las personas fueron contactadas por teléfono y expresaron su interés en compartir características de su vida social, referidas a los momentos de relación en situaciones de padecimiento; los cuidadores formales, en este caso profesionales de la salud, fueron seleccionados por su experiencia clínica, de trabajo interdisciplinario y comunitario para el manejo de las enfermedades crónicas mediante la estrategia de intensidad. Éstos fueron referidos por los coordinadores de los programas de los centros de salud. Una manera de haber constatado su representatividad para el estudio fue mediante el relato de la experiencia laboral, con al menos dos años en la institución. A cónyuges, hijos y vecinos se les identificó, principalmente, como cuidadores informales, en ellos se consideraron las características de criterio definidas por los receptores del cuidado 22 . En todos se aseguró la disponibilidad para acudir a la entrevista colectiva durante el tiempo y lugar convenido.

Referente alas técnicas de recolección delainformación, se elaboró un protocolo para los grupos focales con preguntas semi-estructuradas.

Durante la realización de los grupos focales, la estrategia de comunicación permitió seguir el discurso natural entre los participantes para identificar los elementos propios de la interpretación del significado del capital social. Se realizaron únicamente audio grabaciones de los encuentros con una duración de $120 \mathrm{mi}$ nutos para cada grupo. Todas las entrevistas fueron transcritas y codificadas como parte del procesamiento, organización de la información y recuperación de datos textuales, este proceso es una estrategia analítica que busca encontrar patrones comunes y disímiles en la codificación abierta; el procesamiento de la información se hizo manual; con esta codificación se identifica- ron tres categorías centrales, la primera relacionada con las redes sociales y su influencia en las normas impuestas, la segunda con la confianza, las normas aprobadas y la tercera con los recursos propios para la movilización del ego hacia la conformación de sus redes sociales.

Con la codificación axial 23 se construyeron veinte subcategorías, con la relación entre ellas se diseñó el "paradigma de la codificación" para certificar la conservación "original" de los conceptos planteados por los participantes, dicho de otra manera, se identificaron los niveles para la interpretación y formulación del significado así: el primero relacionado con los códigos, en él se incluyó la información correspondiente al concepto en vivo, el segundo con las interpretaciones, en éste se nombraron y definieron las subcategorías y las categorías. El tercer nivel recogió las reflexiones analíticas, referidas a las interpretaciones contrastadas, entre las categorías y el significado propiamente dicho, que hizo una aproximación teórica de la realidad.

Durante los análisis emergieron 850 códigos en vivo que fueron, posteriormente, integrados en un set de 142 códigos de códigos para el ego (74; 52,11\%), el alter $(47 ; 33,05 \%)$ y frente a la posibilidad de relacionamiento entre ellos (21; $14,78 \%$ ) identificados en los resultados por dos letras mayúsculas y un número.

Las subcategorías relacionadas con el ego fueron definidas como manejo de la enfermedad, soledad, adaptación de la enfermedad, conocimiento y la salud, reconocimiento de la enfermedad, aporte al bienestar, confianza institucional, confianza social, reciprocidad, autoconfianza y la fe. Para el alter se identificaron el rol de la familia, de los profesionales, de los amigos, las actividades voluntarias, de integración social y el soporte social. El sistema de salud y los lugares -el barrio- como contexto; la limitación económica, social y ambiental representada en la inseguridad en el barrio fueron las subcategorías para el alter ego.

El rigor necesario de la investigación cualitativa 24 fue alcanzado desde un enfoque constructivista, mediante la integración variada de técnicas "para oír a la gente y aprender de ella". Con el fin de asegurar la calidad de los datos y sus conclusiones, se realizó triangulación entre los investigadores y se tuvieron en cuenta de los criterios COREQ (Consolidated Criteria for Reporting Qualitative Research), como una lista de verificación que reúne las consideraciones para la presentación de informes de estudios cualitativos 25 .

Con relación a los aspectos éticos, se tuvo en cuenta la actitud de interrogados e investigadores frente a lo investigado, partiendo de la pre- 
misa que en la metodología cualitativa se aplica un gigantesco microscopio a la vida social $26 \mathrm{y}$, por tanto, se permite reconocer que para hacer un uso cuidadoso de la información y lograr la validez científica se debe mantener el anonimato de los participantes, sus opiniones y de ser necesario compartir los resultados, a fin de motivar el esclarecimiento o dudas frente a posturas que fortalezcan futuros debates.

Ninguna de las declaraciones $\mathrm{u}$ opiniones fueron atribuidas a los participantes, todas las personas que aceptaron la invitación firmaron el consentimiento informado. El Centro de Investigación de la Facultad Nacional de Salud Pública de la Universidad de Antioquia, la Empresa Social de Estado METROSALUD y la aseguradora EPS-SURA otorgaron avales éticos.

Se mantuvo el respeto por los interrogados, se omitieron juicios de valor y concepciones previas a la entrevista, a fin de no sobredimensionar los resultados, dándole prioridad en el análisis de los resultados a los contextos de donde provienen las personas e interpretar articuladamente todos los eventos observados y registrados en las transcripciones. Se consideraron tres principios epistemológicos fundamentales, (a) el conocimiento como producción constructiva e interpretativa, y no la suma de hechos definidos por constataciones inmediatas del momento empírico; (b) el proceso de producción de conocimiento como un asunto interactivo y (c) lo legítimo en la producción de conocimiento cualitativo, dado por la singularidad otorgada al significado 27 .

\section{Resultados}

El significado del capital social emerge de la integración entre las categorías y conceptos como vulnerabilidad, exclusión social e inequidades en salud, según la condición de ser hombre o mujer.

La Tabla 1 describe las características de los participantes según género, la Tabla 2 presenta el guion de temas utilizado para entrevistas y grupos focales y la Tabla 3 recoge citas textuales

Tabla 1

Características de los participantes.

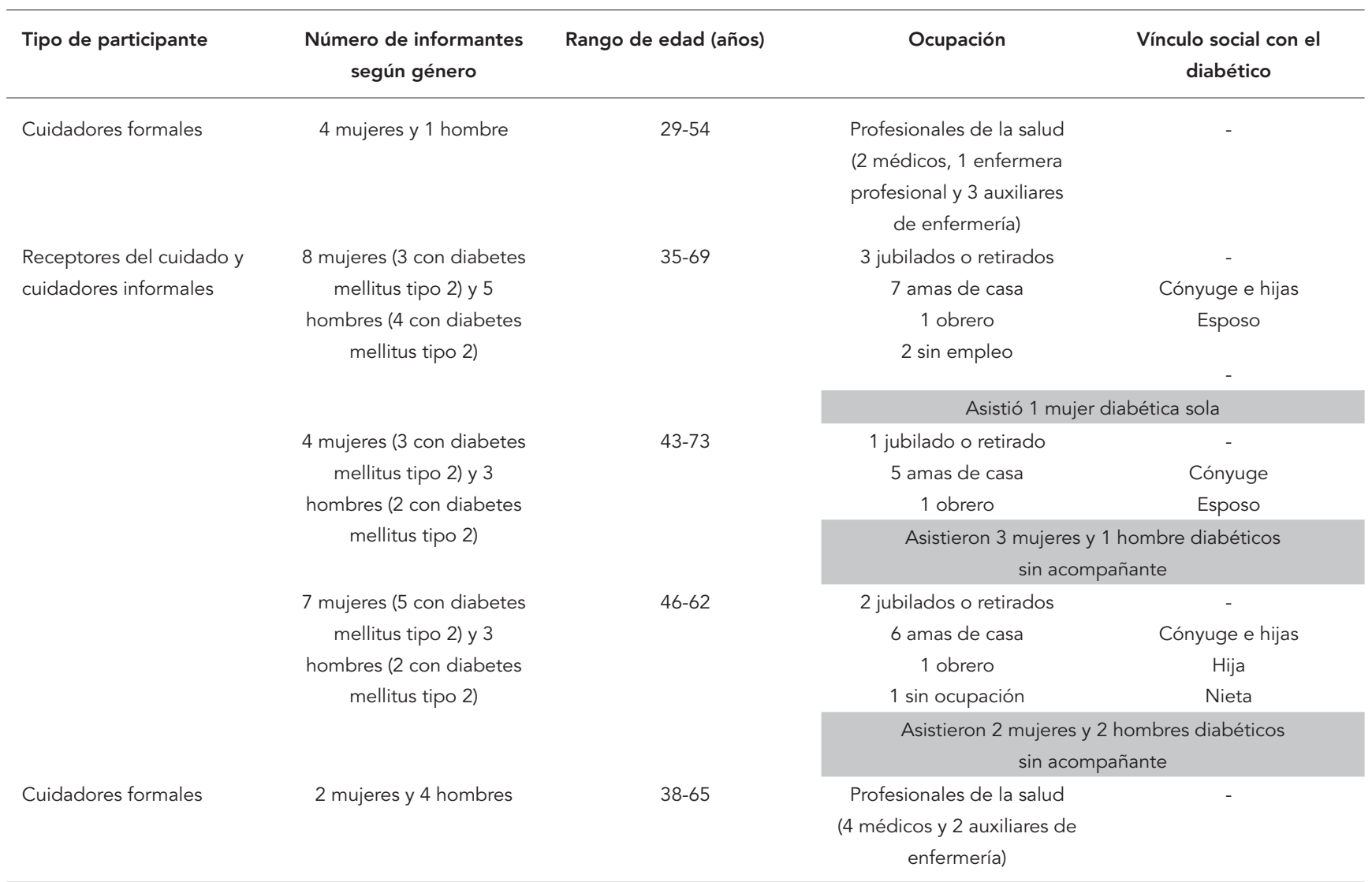




\section{Tabla 2}

Guión de temas utilizado para entrevistas y grupos focales.

\section{Temas}

Contenidos a indagar

Datos sociales y demográficos

Proceso salud-enfermedad de la diabetes mellitus tipo 2

Relación con familiares, amigos, vecinos, compañeros de trabajo, personal de salud

El valor de la confianza para apoyarse en los otros

La participación en actividades sociales y cívicas

Temor a padecer complicaciones

Los hábitos y los riesgos de complicación

Expectativas previas y percepción actual

Perspectivas de futuro

La diabetes mellitus y su relación con el capital social
Sexo, composición familiar (vive solo., actividades que desarrolla en su vida cotidiana, nivel educativo (RC). Sexo, ocupación, tiempo destinado para conocer sobre las enfermedades de riesgo cardiovascular, especialmente la diabetes mellitus tipo 2 (CFI).

Historia del diagnóstico de la diabetes mellitus tipo 2, antecedentes familiares, hábitos saludables, participación de programas de promoción y prevención para riesgo cardiovascular, percepción del estado de salud, uso de medicamentos, información sobre el estado de salud (RC).

Conocimiento de la enfermedad, preocupación por que otras personas conozcan sobre el proceso de cronicidad de la enfermedad (CFI).

Opinión de sus amigos de su enfermedad, hábitos saludables de los amigos, comportamiento en eventos sociales, cuidado de los amigos por la condición de enfermedad, percepción de sentimientos de lástima de los otros, confianza en el personal de salud, información sobre la enfermedad para lograr el auto cuidado de su salud, el control de su enfermedad y sus posibles complicaciones (RC).

Influencia de los amigos y familiares en la evolución de la enfermedad, cuidado de la salud, importancia de relaciones sociales del paciente y hábitos saludables, apoyo de familiares y amigos para sobrellevar la enfermedad, confianza del paciente en instrucciones médicas (CFI).

Sensación de seguridad en la casa, en el barrio, en el lugar de trabajo (RC).

El valor de la confianza depositada de los enfermos en el médico, estrategias para mejorar la confianza en los pacientes diabéticos, pérdida y recuperación de la confianza en los amigos, la familia y los prestadores (CFI).

Conocimiento de actividades programadas para la salud, actividades recreativas realizan en el barrio, descripción de recursos para acudir a un servicio de salud, transporte, policía, solicitud de ayuda a una asociación, la iglesia, a los grupos reconocidos en el barrio, apoyo a las juntas locales de salud, creencia en la veedurías ciudadanas en salud (RC; CFI).

Pensamientos catastróficos por un nuevo diagnóstico de salud, conversaciones, preparación a la familia y amigos sobre complicaciones de la enfermedad (RC).

Cambios en estilo de vida de los pacientes por complicaciones, acompañamiento ante nueva noticia en salud (RF1).

Seguimiento a recomendaciones en alimentación, ejercicio, hábitos de fumar, beber, uso de medicamentos apoyo de la familia para lograr el cambio de dieta, ayuda en rutina con el manejo de medicamentos que debe consumir, uso del tiempo libre (RC).

Mecanismos de aprendizaje para preparar comida, acciones de recuerdo (CFI).

Sentimientos encontrados por manejo de la enfermedad, motivación para seguir adelante (RC). Acciones de vigilancia y control de la enfermedad a partir del trabajo comunitario, liderar procesos comunitarios en personas con enfermedades crónicas no transmisibles, sugerencias para incluir desde su unidad de trabajo y como profesional acciones comunitarias (RF1).

Pensamientos sobre el futuro de la calidad de vida, planes para mejorar la propia calidad de vida y la de los otros, interés en abordar las dimensiones sociales y culturales de la enfermedad y no sólo las clínicas (RC).

Percepción de la salud en caso de tener condiciones económicas, sociales, de seguridad del hogar, en el trabajo adversas en relación con sus amigos (RC).

Percepción de las dificultades de los pacientes, amigos y vecinos diabéticos ante una situación económica desfavorable (CFI).

CFI: cuidador formal e informal; RC: receptor del cuidado; RF1: identifica los elementos para la concepción de la categoría principal relacionada con las redes sociales y su influencia en las normas impuestas y significado. 
Tabla 3

Citas textuales de los participantes correspondientes a las dimensiones de capital social.

\begin{tabular}{|c|c|c|}
\hline Receptores del cuidado & Cuidadores formales & Cuidadores informales \\
\hline Redes sociales & & \\
\hline $\begin{array}{l}\text { "Esa ayuda que la señora dice [vecino], lo que } \\
\text { pasa es que a uno lo primero que lo llaman es pal } \\
\text { almuerzo caliente que llaman, el almuerzo pa' los } \\
\text { de la tercera edad, cuando yo hice esas vueltas me } \\
\text { dijeron, porque yo insistí mucho que en vez del } \\
\text { almuerzo me dieran la ayuda y tal cosa y entonces } \\
\text { gracias a Dios logré y me dijeron 'no, vea es que eso } \\
\text { va por turnos, a pesar de que vaya faltando gente, } \\
\text { vamos... la gente que va falleciendo y tal cosa, se va } \\
\text { escalafonando el turno y ya se les quita el almuerzo y } \\
\text { se les da la ayuda' y yo llegué de verdad a ese punto } \\
\text { donde me llamaron 'bueno no se le va a seguir dando } \\
\text { el almuerzo, pero se le va a seguir dando la ayuda' } \\
\text { y yo en este momento estoy recibiendo la ayuda, } \\
\text { bueno lo otro que yo les quiero decir es... en ese } \\
\text { aspecto yo me siento muy contento porque siento } \\
\text { mucho aprecio de la familia y de los hijos y de los } \\
\text { vecinos, tengo unos vecinos muy buenos donde no } \\
\text { faltan... desde que tengan la oportunidad no faltan } \\
\text { de la casa, así sea conversando con uno, que uno se } \\
\text { entretiene y uno mata el tiempo" (GF5P7C23) }\end{array}$ & $\begin{array}{l}\text { "A lo mejor también se da como a falta } \\
\text { de conocimiento de las redes sociales que } \\
\text { nos rodean a nosotros, o sea si usted me } \\
\text { pregunta en el barrio qué habrá de redes } \\
\text { sociales yo sé que hay un día en que hacen } \\
\text { aeróbicos por allá, pero no sé qué otra cosa } \\
\text { hay para niños, no sé qué cosa hay para } \\
\text { ancianos, no sé, y los medios publicitarios, } \\
\text { radio, televisión, tampoco le informan a uno } \\
\text { nada de eso" (GF4P15C29) }\end{array}$ & $\begin{array}{c}\text { "A mí mi hija me dice 'mamá consígase una } \\
\text { casa que sea de un solo piso' y yo le digo } \\
\text { 'yo de villa hermosa no me voy, vivo cerquita } \\
\text { de la iglesia, cerquita de muchas cosas, yo } \\
\text { conozco la gente, así la gente no lo visite } \\
\text { pero uno se saluda, uno va o ellas van en un } \\
\text { momentico, o la gente lo conoce aunque no } \\
\text { vayan a la casa, o el vecino ve que... bueno } \\
\text { sí, yo salgo confiada, en otra parte no se } \\
\text { puede"' (GF3P30C129) }\end{array}$ \\
\hline
\end{tabular}

\section{Confianza}

“ ¿Sabe también a que debe que la gente no asista? A lo mismo, usted ya sabe lo que le van a decir, entonces ¿pa' qué vamos a volver? Si nos van a decir lo mismo 'no eso yo ya lo sé', uno escucha pues, o sea yo tampoco me gastaría del afán, ¿Pa' qué? Pa' que me digan lo que yo ya se, hay que hacerlo de una manera dinámica, o sea cambiar... cambiar de lugar, cambiar de estrategia, cambiar de programa, de pronto compartir con otras personas de otros barrios..." (GF1P29C100)
"Los pacientes ehh tienen que estar muy enmarcados en la parte social, cultural y económica ehh el tipo de pacientes que manejamos nosotros manejan un grado pues de educación poca, ¿Cierto? Su situación económica es muy vulnerable -demasiado- $y$ su situación de acomodarse como a una normatividad digamos y como a un esquema el cual el profesional tanto de salud, médico, enfermera, nutricionista, trata de darle con lo mejor posible, no sabemos ellos como lo toman, porque ellos no pueden acceder a eso tan fácil como nosotros lo tratamos de decir, porque cuando uno ve la contra respuesta en la consulta 'ay doctor, ah yo trato de hacer eso doctor pero es que en la casa yo tengo que comer allá, yo vivo arrimado, y tengo que comer el sancochito que hagan, está salado o el aguapanelita porque eso es lo único que puedo comer doctor' entonces a pesar de que uno trata de hacer lo máximo eh eh es difícil" (GF4P7C17)
"Pues yo digo que esa confianza que le brindan a uno es como entregarle a uno las llaves y que uno por medio de eso debe corresponderle a aquella persona que le tiene esa confianza y que es como una familia para uno" (GF6P14C65)

(continua) 


\begin{tabular}{|c|c|c|}
\hline Normas & & \\
\hline $\begin{array}{c}\text { "Claro que uno no puede auto medicarse eso es algo } \\
\text { muy grave ¿Cierto? Porque uno no sabe que está } \\
\text { tomando y lo que... lo que le puede servir a ella para } \\
\text { mi puede ser completamente lo contrario, puede } \\
\text { afectarme más ¿Cierto? Lo mejor es si tratar de llevar } \\
\text { una dieta y ser muy consciente de la enfermedad que } \\
\text { se tiene y hacer lo posible por... por... todo está en } \\
\text { la conciencia de que la salud es primordial para todo" } \\
\text { (GF1P24C79) }\end{array}$ & $\begin{array}{l}\text { "Es que es muy difícil en este momento que } \\
\text { ello pues como que se concienticen de eso, } \\
\text { si nosotros mismos que conocemos mucho } \\
\text { los riesgos de la enfermedad y somos 'ah no } \\
\text { el dulcecito, no yo no lo voy a dejar, no es } \\
\text { que, que pereza ¿Cierto?' ahora ellos que ya } \\
\text { venían con esos hábitos y por eso mismo es } \\
\text { que tienen ya la enfermedad crónica, es muy } \\
\text { difícil uno llegar en un momento y decirles } \\
\text { 'tienes que suspender esto, esto' y empezar } \\
\text { a hacer un montón de indicaciones donde } \\
\text { ellos van a decir 'no es que estos van a venir } \\
\text { aquí a cambiarme la vida, ya yo venía así, no, } \\
\text { yo no voy a cambiar pues como mis cosas"' } \\
\text { (GF4P12C26) }\end{array}$ & $\begin{array}{l}\text { "Vea yo le digo pues en mi lugar. Yo no vivo } \\
\text { con mi mamá, yo tengo mi hogar a parte } \\
\text { pero con mi mamá vive una hermana que es } \\
\text { la que mantiene más pendiente de ella, de } \\
\text { su alimentación y de todo eso, por ejemplo, } \\
\text { a mí me puede pasar eso lo que Usted está } \\
\text { diciendo en este momento, que me... relajo, } \\
\text { pero no en el sentido pues de que no me } \\
\text { importa sino que a mi hasta a veces se me } \\
\text { olvida, yo personalmente le llevo cosas que } \\
\text { ella a mí me dice 'Usted sabe que yo no } \\
\text { puedo comer eso' y yo 'ahhss' pero no es por } \\
\text { mal, sino que a mí se me olvida que ella tiene } \\
\text { eso" (GF1P4C16) }\end{array}$ \\
\hline
\end{tabular}

\section{Solidaridad}

"Yo al principio me sentía desanimada, el único que me visitaba era mi hijo cuando podía salir del trabajo, cuando de pronto llega toda esa gallada de allá del barrio, creo que en ellos iba él y la señora, vea me dijo esa muchacha enfermera 'por Dios, usted no me dijo pues que usted no tenía a nadie, y todo ese gentío esperando que los dejemos entrar' y yo 'hay bendito sea que Dios los bendiga' y vea que eso es una cosa bonita y lo alegra a uno porque yo me sentía triste" (GF6P8C38)

\begin{abstract}
"Me parece importante que el paciente se rodee de otros pacientes que también están atravesando por la misma situación, eso ellos pretenden o dicen 'ah bueno este es descuidadito y este terminó en tal o cual complicación y este ve que está más juicioso' y ya les da más moral ¿Cierto? Se animan más en su auto cuidado" (GF4P4C9)
\end{abstract}

"Yo le digo una cosa, a uno porque yo me levanto y lo primero que me preguntan es ¿¿Le traigo las pastillas o ya se las tomó?' y yo 'Ah no me las he tomado', pa' tomarme las pastillas, ¿'Se tomó las pastillas? -no aquí listo', pero él se va a trabajar, ¿Cierto? Ya se van a trabajar los otros dos hijos que tengo, porque tengo otros dos que ya están grandes, ya cada cual tiene su... su familia por aparte, entonces en la casa no vivimos sino cuatro, todos tres se van a trabajar quedo yo sola" (GF3P9C50)

\section{Reciprocidad}

\footnotetext{
"Sí, y eso es lo que a mí más me mata, porque es el único que está viendo por el papá soy yo, los otros hermanos míos no me, no me acompañan, él tiene un problema que él no quiere estar donde la familia, donde los hijos, sino por allá solo donde un familiar lejano por allá, y entonces yo a veces los llamo y salgo es de pelea con ellos, yo los insulto, y les digo de todo, porque es que no me quieren ayudar, entonces eso me está matando a mí, yo se los dije a ellos, un día ustedes van a saber la noticia que yo caí como un pollo muerto ahí listo, porque es que no hay pa' más" (GF6P3C8)
}

"La empatía que se logra es muy grande entre enfermería y médicos pienso yo, es bastante grande, pero es difícil entrar a esa socio cultura del paciente, es difícil, creo yo que se necesita (aunque lo hay.

, se necesita apoyo de enfermería, apoyo de trabajo social y psicología de un equipo

interdisciplinario, ahora que estamos

hablando tanto de salud mental, un equipo que apoye eso" (GF2P5C32)
"Si es que eso depende de la conciencia
de uno, por ejemplo yo he tenido amigos
de que... de que están enfermos que no
pueden trabajar o algo, uno siempre les
hace la visita, le lleva un cariñito, se les da
cualquier cosita y le ayuda" (GF1P32C110)

GF5P7C23: Grupo focal número del 1 al 6; persona número del participante; código número 000-1000.

de los participantes correspondientes a las dimensiones de capital social. La Figura 1 explica la integración de las categorías, y la Figura 2, una aproximación a los aspectos teóricos del capital social encontrados en el estudio.
Para los diabéticos, las relaciones sociales emanan de la estructura social en la que habitan y son el mayor recurso de su capital social para aumentar las destrezas y mejorar las experiencias para el manejo y cuidado de la enfermedad. 


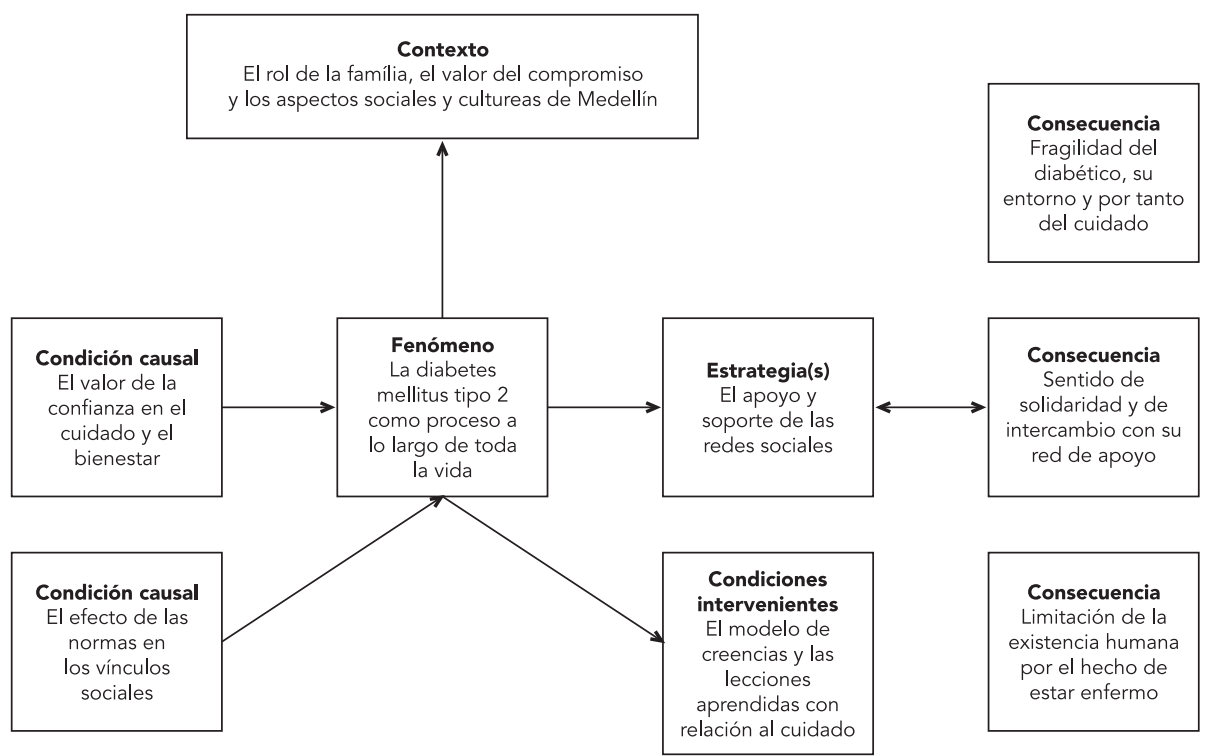

Modelo explicativo sobre la interacción social de percepciones intersubjetivas entorno a la diabetes tipo 2.

En este estudio se le reconoce al capital social, el potencial que tiene en el individuo para que sus redes sociales estén presentes en la consecución de fines para mejorar su situación de salud, para enfrentar entre otras carencias de los grupos primarios, la soledad; sentida más en mujeres que en hombres como una soledad no elegida en la que el individuo siente desprecio, rechazo y nimiedad.

Los pacientes, hombres y mujeres se aíslan por su enfermedad, la soledad los entristece, en general, se sienten solos con su enfermedad, aunque vivan en casa con su familia, sienten que deben preocuparse por sí mismos, porque nadie más lo hará, sin embargo, algunos pacientes manejan la soledad, prefieren no ir a encuentros sociales para no descuidar su salud, sufren cuando los invitan a fiestas, no comen con sus amigos, comen lo que les dan en las reuniones, porque han logrado adaptarse a la enfermedad y viven la salud y la vida aún con las limitaciones que ello implica (AE8).

De otro lado, el cuidado es un asunto de confianza, es un asunto recíproco y solidario de intercambio social; también es cuestión de género. El valor de la confianza siempre está presente en los diferentes espacios donde transcurre la vida en las moradas (hogar, trabajo, espacios habita- dos por personas que se conectan estructuralmente), permitiendo que el cuidado se establezca como una acción de bienestar para la vida. Para los hombres y mujeres participantes el valor de la confianza se define como la respuesta favorable que se encuentra en quien ha depositado seguridad, esperanza o una conducta relevante para el futuro.

La confianza hace que las acciones del cuidado sean más fáciles de realizar, puesto que mejora la comunicación, la reacción ante el manejo y control de la diabetes. La práctica del cuidado se identifica más en mujeres que en hombres, es algo que se da naturalmente y trasciende a la vida colectiva, se relaciona con un tiempo y una cultura, se hace más consciente en mujeres profesionales de la salud que en hombres. El cuidado está determinado por la confianza, dado un marco social e histórico, donde tiene sentido la vida de hombres y mujeres. Las mujeres diabéticas confían en que alguien les puede ayudar, a pesar de no participar de organizaciones barriales (AV4), las mujeres logran usualmente relacionarse con las personas que se encuentren en sus mismas condiciones (CS5), organizan grupos con otros diabéticos para compartir la realización de actividades (AIS2) para trabajar grupalmente por su salud, aunque no saben 
Como EFECTO: la vulnerabilidad, la exclusión social y las inequidades en salud

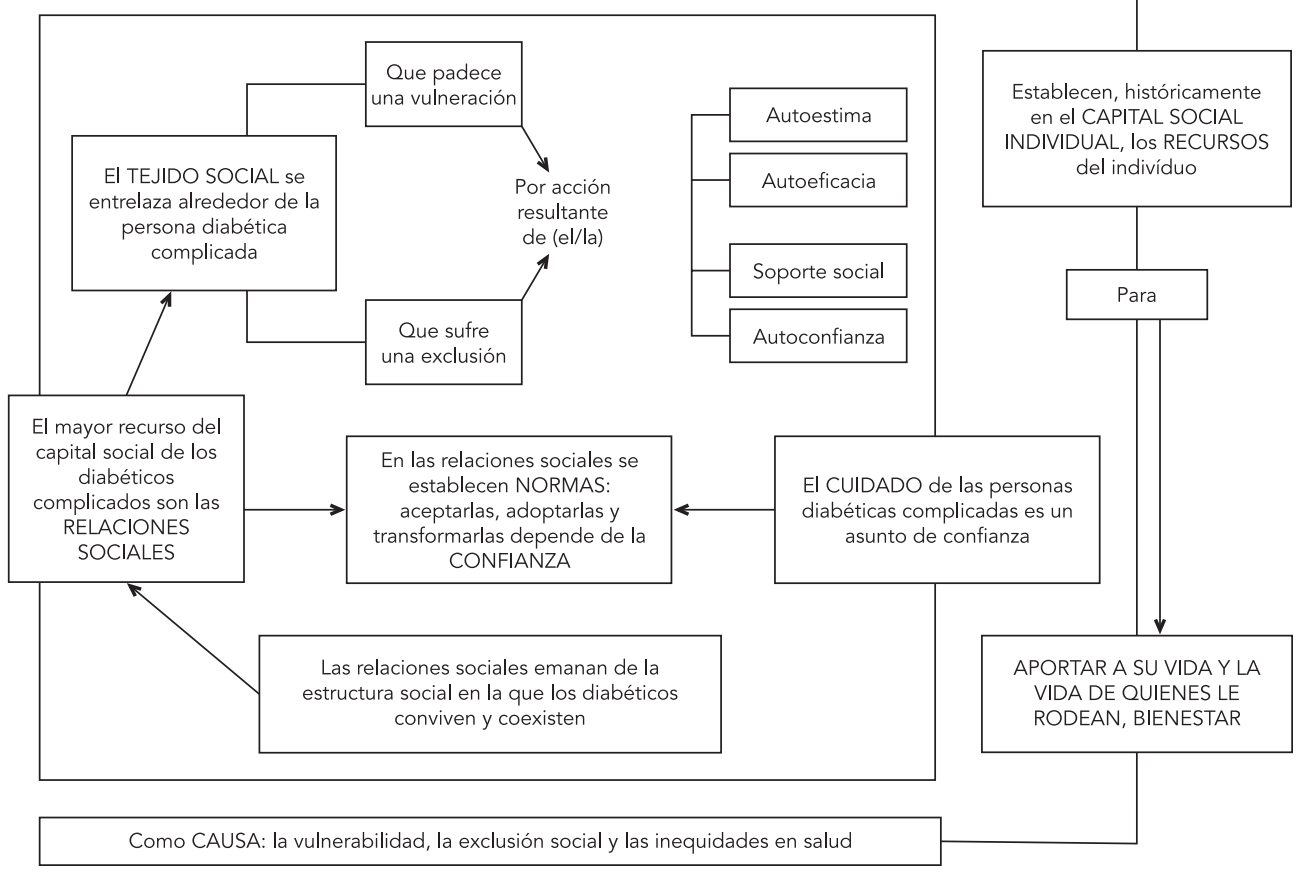

cómo hacerlo, las mujeres se motivan más por liderar estas iniciativas.

Así, la reciprocidad entre los diabéticos se da cuando las instituciones de salud deciden trabajar en red (R5), a pesar de la inseguridad marcada en algunos sectores de la ciudad de que les impide caminar y salir al encuentro del otro (SB1), siendo aún más grave cuando los conflictos sociales le impiden al profesional de la salud movilizarse por la ciudad asistiendo a los pacientes (SB3).

Finalmente, el tejido social se entrelaza alrededor de la persona diabética que padece una vulneración por acciones resultantes de su autoconfianza, el soporte social autoeficaz y la autoestima del mismo en circunstancias en la vida que, indirectamente, contribuyen con el deterioro del capital social; entre ellas están el aislamiento, la búsqueda de respuestas curativas en la religión o la desmotivación por el auto cuidado.

El estrés, la tristeza, los problemas familiares deprimen y descompensan a los pacientes (AC1), los excluye, vulnera su autoestima como también los momentos de enojo (AC2) que bien pudieran replicarse en otros padecimientos crónicos, aunque la depresión no fue una estudiada en profundidad, uno de los momentos de mayor tristeza que señalan los pacientes es reconocerse como diabéticos diagnosticados (RE1). Nada diferente a lo que sucede con las enfermedades agudas, frente a los sentimientos que se desatan de rechazo, negación, temor a la muerte, tristeza (RE2) y que en la condición humana están presentes ante la pérdida del bienestar.

El paciente motivado por salir adelante nunca pierde la esperanza (AC4), no le da vergüenza definirse como diabético, ayuda a otros, se siente seguro, no minimiza sus actuaciones, se siente útil y servicial, permitiéndose tejer sus relaciones sociales y nutrirlas con nuevas actividades, la religión cumple un rol importante dentro de la vida de las mujeres con actitudes de mayor autoconfianza, los adultos mayores creen que el "mejor doctor" es Dios (F1). Los pacientes se ponen en las manos de su Dios para que los ayude a llevar su enfermedad y se resignan a su voluntad (F2). De alguna manera, los pacientes buscan apoyo emocional y social más que apoyo científico y 
clínico, les gusta ser escuchados, desean compartir sus experiencias con otras personas y acuden a Dios en todo momento (SS1).

\section{Discusión}

A pesar de las limitaciones en el uso del concepto para la salud, la indagación del significado del capital social proporciona elementos para la apropiación de categorías, como las relacionadas a los intercambios sociales en el bienestar, éstas como parte del proceso de reconocer la existencia de las redes sociales, frente al padecimiento de una patología que seguramente empeorará 28 .

No cabe duda que la enfermedad misma limita la existencia humana, se interpone a la vida del hombre, frena sus actividades, proyectos e ilusiones, condiciona su quehacer, limita el sentido que le da a la vida. Así las cosas, el concepto está ligado al campo de la política para la salud pública, cada vez que se vuelve la mirada hacia los modelos de atención, respecto a las necesidades del individuo, se recuerda que siempre se está en desventaja social por estar enfermo, las desventajas en salud disminuyen las posibilidades hasta de conservar la salud, lo hacen más susceptible a las injusticias sociales, relacionadas directamente hasta con la forma desigual de distribuir los recursos financieros y sociales 29. Los modelos de salud no deben responder únicamente a una gestión basada en resultados de operación para la salud, sino a la producción de salud centrada en las personas.

En este contexto urbano, las desigualdades en salud son evidentes por la posición social de los pacientes, las instituciones 30 , pero sobre todo por políticas, económicas y jurídicas que prevalecen en la vulneración al derecho a la salud, porque la lucha del paciente no es per se por la pobreza, sino por las estructuras sociales que la contienen.

Es claro que el capital social "individual" como recurso que existe en conjunto entre los individuos es productivo y ayuda a los individuos en el logro de sus objetivos, señalando que es más común encontrar indicadores de contexto que de los individuos, por eso Coleman 31 describe el capital social como inherente a la estructura de las relaciones entre los actores y entre actores para referirse a los aspectos de la sociedad que hacen más fácil la toma de decisiones de un conjunto de personas, pero a su vez incluye las obligaciones, las expectativas y la credibilidad de las personas para producir logros eficaces. En esta investigación la denominación de capital social "individual" se plantea desde la teoría de generación de recursos sociales para el acceso y movili- zación de capital social como dos aspectos que implican, como las dos caras de una moneda, usar los recursos, pero también devolverlos 9,10.

El capital social "individual" se adapta a la estructura social 31. Las mujeres diabéticas son más visibles en las redes de relaciones a las que pertenecen que los hombres; a través de las redes activan recursos -con y sin conocimiento del concepto de salud- para lograr estrategias en el manejo de la enfermedad. Este es el concepto en el que Coleman insiste como base principal del capital social y no necesariamente en la confianza fundada, claramente establecida por la cultura, como lo afirman Putnam 32 y Fukujama 33.

Aunque a las personas que padecen diabetes se les indaga por el logro de metas individuales (calidad en la atención del servicio de salud, mejora en la alimentación, el control de sus hábitos), pocas veces se hace sobre las situaciones relacionadas con los afectos. Como es bien sabido, el intercambio de recursos 34 incluye sentimientos contrarios, de aislamiento, aflicción, soledad, nostalgia, tristeza, desamparo, etc., el reconocer los recursos sociales del individuo como estrategias de integración a los colectivos pudiera no sólo incorporarlo en entornos saludables como ámbito de desarrollo, sino en el quehacer cotidiano en medio de la adversidad 35 , y así mejorar los esquemas planteados en los modelos de atención, por ejemplo, el modelo de cuidado crónico 36.

En esta investigación no se enfatizó en los recursos institucionales para la salud, sino en los vínculos que las personas crean según su entorno para el desarrollo de la vida en actividades de relación, reproducción y participación social 37, que no es otra cosa que crear nuevos marcos de fortalecimiento social para superar realidades, tanto de la persona, como de la comunidad en sus estructuras familiares mediante reconstrucción de los valores sociales y reintegración de los cambios en la sociedad que le circunda 38,39.

Ampliar la noción de este significado contribuye al conocimiento de las dinámicas sociales contextuales para afrontar un padecimiento que depende de las acciones e interacciones entre los sujetos y de los recursos propios de un individuo como elementos para la reificación de estructuras sociales, como son los modelos de atención 40.

En este sentido, aun cuando el concepto de capital social, en general, crea tanta controversia 41, es innovadora la perspectiva "individual" para la gestión sanitaria de los servicios de salud, principalmente, para el control de enfermedades degenerativas crónicas como la diabetes, puesto que asume en el individuo su capacidad para de acceder e intercambiar recursos que le son propios con su red social mediante activación de estrategias útiles y necesarias para modelos de 
atención integradores de las necesidades de los usuarios. En otras palabras, para los equipos de atención primaria, introducir aspectos relacionados con los determinantes relacionales de la salud en el seguimiento y control de la enfermedad propone retos de alto interés e impacto en los procesos de reforma de los sistemas de salud en lo relacionado al cierre de brechas de una mejor atención en salud.

\section{Conclusión}

El capital social se considera un determinante de la salud, cuyo efecto puede ser útil en la gestión de políticas dirigidas a mejorar la eficiencia y reducir las inequidades en salud. La acumulación de capital social en los individuos se relaciona con mejores condiciones de salud. Las redes sociales funcionan bajo normas, reciprocidad y responsabilidad, fortalecen los lazos personales, familiares y comunitarios.

La participación es mayor en mujeres que hombres mejorando las condiciones de vida de grupos y personas a través de la confianza, la solidaridad y la reciprocidad lo que hace pertinente fortalecer estrategias de atención, a partir del reconocimiento de los recursos individuales de capital social en su entorno.

El manejo de la diabetes tipo 2 demanda de procesos integrales que no están en manos sólo del prestador de salud, si bien la confianza en el médico es fundamental, la gestión integral de las acciones preventivas una vez se han establecido entidades complejas dependen primordialmente del paciente, de su propia motivación, autoconfianza, autoestima y autoeficacia, quien ayudado por el prestador es capaz de tomar control de la enfermedad junto con su familia y en el mejor de los casos por las redes facilitadas para el uso de los servicios de salud y de los recursos comunitarios, con el objeto de lograr perfiles de riesgo monitoreables en entornos sociales que no siempre le son favorables.

La diabetes tipo 2 comparte factores de riesgo con otras enfermedades crónicas, acciones similares y dirigidas a los mismos grupos de población, los hallazgos de esta investigación interesan de manera amplificada a los programas de asistencia dirigidos a controlar en general, los riesgos cardiovasculares por la importancia que tienen las redes sociales y otros mecanismos eficaces proporcionados por el capital social, puesto que, este al ser creado por las interrelaciones que se dan entre las personas, contribuye en la comprensión psicosocial del padecimiento crónico.
De otro lado, a pesar de la existencia de evidencia epidemiológica del capital social en salud, su estudio con enfoques cualitativos genera estrategias de promoción de la salud y prevención de la enfermedad en las personas y los lugares donde transcurre su vida y asume una apropiación endógena del sufrimiento señalando la importancia de las redes sociales, las normas y la confianza para el cuidado esencial de las complicaciones derivadas de la diabetes, en tanto ninguna persona puede existir como un ser humano de forma aislada, máxime cuando un padecimiento crónico obliga al individuo a comprender, aceptar y adaptarse a lo largo de la vida de la condición biológica de deterioro y social de afrontamiento.

En este estudio el conocimiento generado para el manejo y control de los diabéticos 2 desde la perspectiva "individual" del capital social es considerado más que una variable instrumental e incorpora categorías de análisis que en términos de política indican que, el capital social "individual" genera maneras eficaces de mejorar las condiciones de salud y debiera ser incluido como el punto de partida para logros de eficacia colectiva en salud pública, dado que tendría un doble efecto, en la persona y en la comunidad, no sólo por la inclusión en los modelos de atención para las enfermedades crónicas de países en procesos de reforma a los sistemas de salud, sino por la integración de características propias de la persona que se hacen relacionales en la intervención social de la historia social de la enfermedad en los que las redes sociales pudieran ser integradoras en la implementación de redes de servicios.

En últimas, la salud de las personas y su bienestar puede verse afectado por su propio capital social "individual" y aunque es escasa la literatura que explica su rol en las inequidades en salud y las diferencias entre grupos sociales, se debe mantener la motivación de investigación sobre el tema toda vez la adaptación y nuevas formas de comprensión del concepto.

Así las cosas, en contextos urbanos similares, marcados por la violencia, altas condiciones de pobreza, pocas posibilidades para el desarrollo humano integral, el capital social "individual” denotará una especial diferenciación dados los aspectos culturales y sociales de la sociedad a la que se debe, por tanto, acepciones para la comparabilidad solo serán posibles si y solo sí se reconozcan elementos validadores como los encontrados en este estudio para el apoyo social y la confianza, principalmente. 


\section{Resumo}

Compreender o significado do capital social, diabetes tipo 2 por sexo, um contexto urbano da Colômbia. pesquisa qualitativa do interacionismo simbólico. 25 mulheres e 16 homens, diabéticos, familiares, vizinhos e cuidadores participaram seis grupos focais. 850 códigos se que foram integrados em um conjunto de 142 codes para o ego, $o$ alter $e$ alter ego. Três categorias $e$ vinte subcategorias foram identificados para o projeto de "codificação de paradigma". O significado não é o mesmo para homens e mulheres. Laços sociais das redes sociais criadas diariamente pela confiança e solidariedade são valorizados cuidado diferente, porque as experiências sociais e fatos resultantes da auto-confiança, auto-eficácia e de apoio social, principalmente, auto-gestão e controle em relação a doença. Os recursos sociais de um indivíduo são reificadas para a gestão o cuidado da doença como uma estratégia para reduzir as desigualdades na saúde.

Diabetes Mellitus Tipo 2; Rede Social; Desigualdades em Saúde

\section{Colaboradores}

E. Martínez-Herrera participó en la concepción, diseño del trabajo, recolección, análisis e interpretación de la información, escritura y aprobación de la versión final del artículo. O. Moreno-Mattar contribuyó com la recolección, análisis e interpretación de la información y aprobación de la versión final del artículo. R. V. H. Dover contribuyó de la concepción del diseño del trabajo, análisis e interpretación de la información, escritura y aprobación de la versión final del artículo.

\section{Agradecimientos}

A los grupos de investigación, Epidemiologia de la Facultad Nacional de Salud Pública (Estrategia de sostenibilidad 2013-3014) y Recursos Estratégicos, Región, y Dinámicas Socio ambientales del Instituto de Estudios Regionales de la Universidad de Antioquia y a la Empresa Social del Estado METROSALUD por su apoyo académico y científico, así como a las instituciones, Empresa Promotora de Salud EPS SURA, Caja de Compensación Familiar COMFAMA y DINAMICA IPS por su apoyo técnico.

\section{Referencias}

1. Castaño A. Modelos de atención para diabetes: experiencias ideales reales posibles. Observatorio de Diabetes de Colombia. Bogotá: Novo Nordisk; 2012.

2. Moscovici S, Hewstone M. De la ciencia al sentido común. In: Moscovici S, editor. Psicología social. Tomo II. Barcelona: Paidós; 1986. p. 679-710.

3. Smith KP, Christakis NA. Social networks and health. Annu Rev Sociol 2008; 34:405-29.

4. Ministerio de Salud y Protección Social. Conjunto de acciones para la reducción multifactorial de las enfermedades crónicas no transmisibles. http:// www.col.ops-oms.org/prevencion/carmen.asp (accedido el 19/Sep/2009).
5. Moore S, Haines V, Hawe P, Shiell A. Lost in translation: a genealogy of the "social capital" concept in public health. J Epidemiol Community Health 2006; 60:729-34.

6. Patussi MP, Moysés SJ, Junges JR, Sheiham A. Capital social e a agenda de pesquisa em epidemiologia. Cad Saúde Pública 2006; 22:1525-46.

7. Organization for Economic Co-operation and Development. The well-being of nations: the role of human and social capital. Paris: OECD Publications; 2001.

8. Rostila M. Social capital and health inequality in European Welfare States. London: Palgrave MacMillan; 2013. 
9. van der Gaag M, Snijders T. Proposals for the measurement of individual social capital. In: Völker HFB, editor. Creations and returns of social capital. London: Routledge; 2005. p. 199-218.

10. van der Gaag M, Webber M. Measurement of individual social capital. Questions, instruments, and measures. In: Kawachi I, Subramanian SV, Kim D, editors. Social capital and health. Boston: Springer Science; 2008. p. 29-49.

11. Pomagalska D, Putland C, Ziersch A, Baum F, Arthurson K, Orchard L, et al. Practical social capital: a guide to creating health and wellbeing. Canberra: National Library of Australia; 2009.

12. Sapag JC, Kawachi I. Social capital and health in Latin America: ecological and individual level analysis. World Med Health Policy 2010; 2:285-99.

13. Jha V, Garcia Garcia G, Iseki K, Zuo L, Naicker S, Plattneer B, et al. Chronic kidney disease: global dimension and perspectives. Lancet 2013; 382: 260-72.

14. Rojas PP. De viaje con Wittgenstein: en busca del significado y la intención. Convivium 2007; 20:197-222.

15. Anscombre JC. Semántica y léxico: topoï, estereotipos y frases genéricas. Revista Española de Lingüistica 1995; 25:297-310.

16. Arcila Mendoza PA, Mendoza Ramos YL, Cañon Ortiz OE, Jaramillo JM. Comprensión del significado desde Vygotsky, Bruner y Gergen. Revista Diversitas - Perspectivas en Psicología 2010; 6:37-49.

17. Moore S, Shiell A, Hawe P, Haines VA. The privileging of communitarian ideas: citation practices and translation of social capital into public health research. Am J Public Health 2005; 95:1330-7.

18. Daza A. Experiencias de Intervención en el conflicto urbano. Tomo I. Medellín: Alcaldía de Medellín; 2001.

19. Isaac J. Erving Goffman y la microsociología. Barcelona: Editorial Gedisa; 1999.

20. Macinko J, Starfield B. The utility of social capital in research on health determinants. Milbank Q 2001; 79:387-427.

21. Portes A. Social capital: its origins and applications in modern sociology. Annu Rev Sociol 1998; 24: 1-24.

22. Vásquez Navarrete ML, Ferreira da Silva MR, Mogollon Pérez AS, Fernández de Sammamed MJ, Delgado Gallego ME, Vargas Lorenzo I. Introducción a las técnicas cualitativas de investigación aplicadas en salud. Cali: Programa Editorial Univalle; 2011.

23. Coffey A, Atkinson P. Los conceptos y la codificación. In: Coffey A, Atkinson P, editors. Encontrar el sentido a los datos cualitativos Estrategias complementarias de investigación. Medellín: Editorial Universidad de Antioquia; 2003. p. 31-63.

24. Ávila MG. Aspectos éticos de la investigación cualitativa. Revista Iberoamericana de Educación 2002; 29:85-103.
25. Tong A, Sainsbury P, Craig J. Consolidated criteria for reporting qualitative research (COREQ): a 32 -item checklist for interviews and focus groups. Int J Qual Health Care 2007; 9:349-57.

26. Cerillo Vidal JA. El intermediario imposible. Algunas reflexiones en torno a epistemología y ética en la investigación cualitativa. Nómadas Revista Crítica de Ciencias Sociales y Jurídicas 2009; 24: $1-15$.

27. Barrio-Cantalejo IM, Simón-Lorda P. Problemas éticos de la investigación cualitativa. Med Clin (Barc) 2006; 126:418-23.

28. Beaglehole R, Yach D. Globalisation and the prevention and control of non-communicable disease: the neglected chronic diseases of adults. Lancet 2003; 362:903-8.

29. Gwatkin DR. Desigualdades sanitarias y salud de los pobres: ¿Qué sabemos al respecto? ¿Qué podemos hacer? Bull World Health Organ 2000; 78:3-18.

30. García Londoño JD, Moreno Mattar O. El capital social y el Sistema de Seguridad Social en Salud en Medellín, 2009. Revista Salud Pública de Medellín 2011; 5:35-55.

31. Coleman JS. Social capital in the creation of human capital. AJS 1998; 94:95-120.

32. Putnam RD. Making democracy work: civic traditions in modern Italy. Princeton: Princeton University Press; 1993.

33. Fukujama F. The great disruption: human nature and the reconstruction of social order. New York: Free Press; 1999.

34. Cohen S. Social relationships and health. Am Psychol 2004; 59:676-84.

35. Krieger N, Davey-Smith G. "Bodies count," and body counts: social epidemiology and embodying inequality. Epidemiol Rev 2004; 26:92-103.

36. Coleman K, Austin BT, Brach C, Wagner EH. Evidence on the chronic care model in the new millennium. Health Aff (Millwood) 2009; 28:75-85.

37. Rocco L, Suhrcke M. Is social capital good for health? A European perspective. Copenhagen: WHO Regional Office for Europe; 2012.

38. Sen A. Desarrollo y libertad. Bogotá: Planeta; 2000.

39. Eriksson M, Emmelin M. What constitutes a health enabling neighborhood? A grounded theory situational analysis addressing the significance of social capital and gender. Soc Sci Med 2013; 97:112-23.

40. Epping Jordan J, Pruitt S, Bengoa R, Wagner EH. Improving the quality of health care for chronic conditions. Qual Saf Health Care 2004; 13:299-305.

41. Kawachi I. Introduction. In: Kawachi I, Takao S, Subramanian S, editors. Global perspectives on social capital and health. New York: Springer; 2013. p. 1-21.

Recibido el 22/Sep/2013

Versión final presentada el 23/Oct/2014

Aprobado el 10/Nov/2014 